\title{
Estudo da miscibilidade das misturas de PHB e PLA, com um PHB de alta polidispersividade
}

\section{Miscibility study of PHB and PLA mixtures, using a PHB with high polydispersity}

\author{
Wagner Mauricio Pachekoski ${ }^{*}$, Carla Dalmolin² e José Augusto Marcondes Agnelli ${ }^{3}$ \\ ${ }^{1}$ Universidade Federal de Santa Catarina - UFSC, Joinville, SC, Brasil \\ ${ }^{2}$ Departamento de Química, Universidade do Estado de Santa Catarina - UDESC, Joinville, SC, Brasil \\ ${ }^{3}$ Departamento de Engenharia de Materiais, Universidade Federal de São Carlos - UFSCAR, \\ São Carlos, SP, Brasil \\ *pwmp1583@gmail.com
}

\section{Resumo}

Neste trabalho, foi estudada a miscibilidade de misturas de um PHB constituído de frações de alta e baixa massa molar com um PLA de alta massa molar. Os materiais extrudados foram analisados pelas técnicas de calorimetria exploratória diferencial (DSC), espectroscopia dielétrica (DE) e análises dinâmico-mecânicas (DMA). A partir dos resultados observou-se o distinto comportamento cristalino das frações de PHB, assim como a miscibilidade parcial do PLA com o PHB de baixa massa molar.

Palavras-chave: PHB, PLA, misturas poliméricas, polímero biodegradável.

\section{Abstract}

In this work, the miscibility of a PHB formed by high and low molar mass fractions and a low molar mass PLA was studied. The extruded materials were analyzed by differential scanning calorimetry (DSC), dielectric spectroscopy (DS) and dynamic mechanical analysis (DMA). From these results, it was observed an unique behavior from the PHB fractions, and the partial miscibility of PLA onto low molar mass PHB.

Keywords: PHB, PLA; blends, biodegradable polymer.

\section{Introdução}

O poli (hidroxibutirato) - PHB é um polímero da classe dos poliésteres, biodegradável de alta cristalinidade e propriedades mecânicas similares ao polipropileno ${ }^{[1,2]}$. Pode ser produzido a partir de fontes renováveis, como a cana-de-açúcar, através de um processo de fermentação bacterial $^{[3,4]}$. Suas propriedades físicas já foram amplamente estudadas através de técnicas como a difração de raios- $x$ de alto ângulo ${ }^{[5-7]}$ e espectroscopia dielétrica ${ }^{[8-11]}$.

O poli (ácido lático) - PLA, assim como o PHB, é um poliéster biodegradável, produzido por síntese química a partir de fontes renováveis como o milho ${ }^{[12-14]}$. Por possuir excelente biocompatibilidade com o corpo humano, é motivo de vários estudos na área biomédica ${ }^{[15-17]}$, como de implantes, tendo suas propriedades físicas bem detalhadas na literatura ${ }^{[13,14,18,19]}$.

Misturas de PHB e PLA de alta massa molar são imiscíveis mesmo na fusão destes dois materiais ${ }^{[20-22]}$. Porém, estudos recentes ${ }^{[23,24]}$ mostraram que misturas de PLA de alta massa molar com PHB de massa molar inferior a $20.000 \mathrm{~g} / \mathrm{mol}$ apresentaram-se miscíveis, com propriedades intermediárias entre ambos os polímeros. Desta maneira, buscou-se estudar o comportamento de misturas de PLA e PHB, utilizando-se um poli (hidroxibutirato) de alta polidispersividade, contendo frações de alta e baixa massa molar, com o objetivo de avaliar a miscibilidade das misturas de poli (hidroxibutirato) e poli (ácido lático).

\section{Materiais e Métodos}

O poli (hidroxibutirato) utilizado neste trabalho foi produzido pela PHB Industrial S/A, através do processo fermentativo FE 60-3. Este material possui dois máximos na distribuição de massa molar: um correspondente a um polímero de alta massa molar $\left(\mathrm{com} \mathrm{M}_{\mathrm{w}}\right.$ de $\left.710.000 \mathrm{~g} / \mathrm{mol}\right) \mathrm{e}$ uma fração de baixa massa molar (com $\mathrm{M}_{\mathrm{w}}$ de $\left.50.000 \mathrm{~g} / \mathrm{mol}\right)$ correspondente a $7 \%$ do peso total. Devido a estas duas frações, a polidispersividade deste lote de PHB é igual a $3,4^{[25]}$. O poli (ácido lático) utilizado para as misturas com PHB foi produzido pela Mitsui Chemical, possuindo uma massa molar com $\mathrm{Mw}=167.000 \mathrm{~g} / \mathrm{mol}$. O PLA 
apresenta uma distribuição de massa molar com um valor de polidispersividade igual a $1,67^{[26]}$.

\subsection{Preparo das amostras}

As amostras dos homopolímeros PHB e PLA foram processadas em extrusora modular, de dupla rosca, co-rotativa Werner Pfleiderer ZSK 30 na forma de grânulos. As misturas poliméricas de PHB e PLA foram preparadas a partir dos homopolímeros não processados, através da co-alimentação em extrusora, e nas seguintes proporções: (PHB/PLA) 75/25, 50/50 e 25/75. A Tabela 1 apresenta as condições de processamento.

Filmes de PHB para as medidas dielétricas foram obtidos a partir da dissolução em clorofórmio a temperatura ambiente, deposição da solução em placa de Petri com subsequente evaporação do solvente, e secagem por uma hora em estufa com circulação a ar numa temperatura de $40^{\circ} \mathrm{C}$. Os demais filmes para medidas dielétricas foram preparados por prensagem a quente. A amostra foi inicialmente aquecida até uma temperatura de $190^{\circ} \mathrm{C}$, com uma pressão de $50 \mathrm{~kJ}$ e com duração de um minuto, sendo a seguir realizado um resfriamento com água até temperatura ambiente.

\subsection{Caracterização das amostras}

As medidas de Calorimetria Exploratória Diferencial (DSC) foram realizadas em um aparelho Perking Elmer DSC7L. As medidas foram realizadas em uma faixa de temperatura de $-55^{\circ} \mathrm{C}$ a $200^{\circ} \mathrm{C}$ em dois ciclos simultâneos, com uma taxa de aquecimento de $20^{\circ} \mathrm{C} /$ minuto.

Os experimentos de relaxação dielétrica foram realizados numa faixa de temperatura entre $-150^{\circ} \mathrm{C}$ e $150^{\circ} \mathrm{C}$. Medidas do domínio de frequência das partes real e imaginária da função dielétrica foram realizadas de $1 \times 10^{-1} \mathrm{~Hz}$ a $1 \times 10^{7} \mathrm{~Hz}$ com um Concept Twelve System
Novocontrol GmbH integrado a um Analisador de Impedância Solartron SI 1260 e uma célula de medida.

As análises dinâmico-mecânicas foram realizadas através de um aparelho DMA Perkin Elmer, de $-30^{\circ} \mathrm{C}$ a $70^{\circ} \mathrm{C}$, numa frequência de $1 \mathrm{~Hz}$.

\section{Resultados e Discussão}

\subsection{Calorimetria Diferencial de Varredura (DSC)}

As Figuras 1 e 2 apresentam as curvas DSC de aquecimento do PHB, PLA e suas misturas (primeiro e segundo ciclo de aquecimento, respectivamente). Observa-se através destes resultados que, tanto para o PHB como para o PLA, são detectadas as suas temperaturas de transição vítrea $(\mathrm{Tg})$, temperaturas de cristalização a frio (Tcc) e temperatura de fusão (Tm). Observa-se ainda que todos estes processos são detectados nas misturas de $\mathrm{PHB}$ e PLA, podendo, em algumas situações, estarem sobrepostos. A Tabela 2 apresenta os valores de todas as situações e os casos de sobreposição.

De acordo com os resultados encontrados, observa-se uma imiscibilidade do PHB e do PLA na mistura, justificada pela presença de duas temperaturas de transição vítrea e dois picos de fusão. Porém, os resultados são contraditórios pelos valores de Tg do PLA nas misturas, onde se observa uma redução na $\mathrm{Tg}$ proporcional ao aumento da porcentagem de PHB, sugerindo uma miscibilidade parcial. Contudo, a mesma variação não ocorre para o PHB. O gráfico da variação da temperatura de transição vítrea do PHB e do PLA nas suas misturas, é visualizado na Figura 3.

Segundo Koyama e Doi ${ }^{[21]}$, misturas de PHB e PLA em que ambos os componentes possuem massa molar acima de $20.000 \mathrm{~g} / \mathrm{mol}$, a temperatura crítica de solubilidade (UCST) situa-se em $5100 \mathrm{~K}$, indicando que as misturas poliméricas de PHB e PLA apresentarão separação de fase

Tabela 1. Condições de processamento das amostras em extrusora.

\begin{tabular}{|c|c|c|c|c|c|c|c|c|c|}
\hline \multirow{2}{*}{$\begin{array}{c}\text { Rotação } \\
\text { (RPM) }\end{array}$} & \multirow[t]{2}{*}{ Torque (\%) } & \multirow{2}{*}{$\begin{array}{l}\text { Pressão } \\
\text { (Psi) }\end{array}$} & \multicolumn{7}{|c|}{ Temperatura $\left({ }^{\circ} \mathrm{C}\right)$} \\
\hline & & & $\mathrm{Z1}$ & $\mathrm{Z2}$ & $\mathrm{Z3}$ & $\mathrm{Z4}$ & $\mathrm{Z5}$ & cabeçote & fundido \\
\hline 200 & 55 & 400 & 170 & 170 & 169 & 169 & 165 & 165 & 165 \\
\hline
\end{tabular}

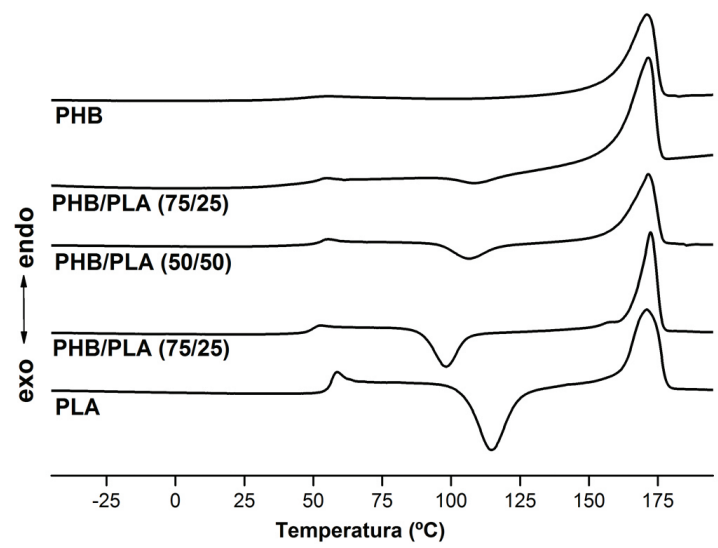

Figura 1. Curva do primeiro ciclo de aquecimento do PHB, PLA e suas misturas através de Calorimetria Exploratória Diferencial (DSC).

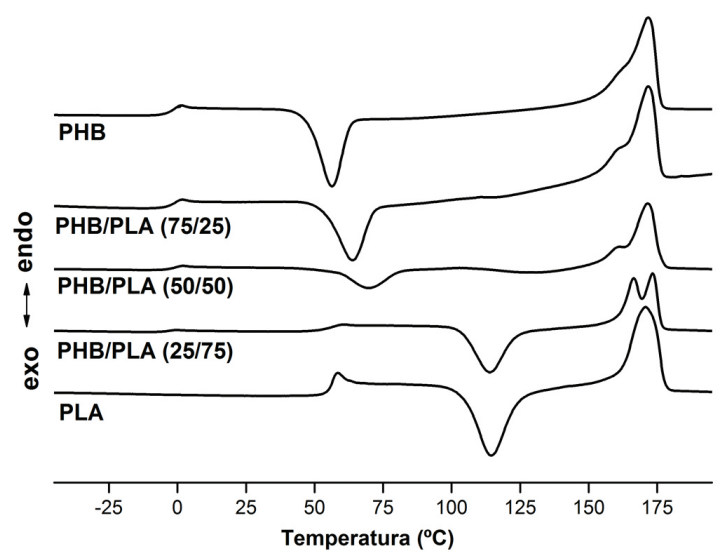

Figura 2. Curva do segundo ciclo de aquecimento do PHB, PLA e suas misturas através de Calorimetria Exploratória Diferencial (DSC). 
na temperatura de fusão, situada na região de $473 \mathrm{~K}$, ou em temperaturas inferiores. Porém, frações de PHB com massa molar menor que 20.000 apresentam solubilidade em PLA na temperatura de fusão dos polímeros ${ }^{[27-29]}$. Como o PHB utilizado neste trabalho apresenta uma pequena fração de baixa massa molar, a mesma pode estar sendo solubilizada e agindo como plastificante no poli (ácido lático), levando a uma redução na temperatura de transição vítrea.

\subsection{Espectroscopia Dielétrica Complexa (DE)}

A Figura 4 apresenta a representação gráfica das relaxações dielétricas do PHB, enquanto na Figura 5, estas medidas são visualizadas em frequência constante (medidas isocrônicas). Nesta medida, foi utilizado um filme de PHB preparado por dissolução em clorofórmio, com o objetivo de evitar que a degradação térmica do polímero influenciasse os resultados.

$\mathrm{O}$ PHB apresenta duas relaxações principais ${ }^{[9]}$. Ambos os processos aparecem como máximos em medidas de perda dielétrica $\left(\varepsilon^{\prime \prime}\right)$, sendo denominados $\beta$ e $\alpha$ em ordem de aparecimento em temperatura crescente. A relaxação denominada $\beta$, é atribuída a movimentos locais de grupos carboxílicos e rotações locais ao redor da ligação C-O em poliésteres. A relaxação denominada $\alpha$, é atribuída à temperatura de transição vítrea do poli (hidroxibutirato). Ambos os processos são determinados na medida de relaxação dielétrica realizada para o $\mathrm{PHB}$. A relaxação $\beta$ é observada a partir de $-50^{\circ} \mathrm{C}$, enquanto a relaxação $\alpha$ é observada a partir de $0^{\circ} \mathrm{C}$. A intensidade e a forma dos dois processos são distintas, sendo a relaxação $\alpha$ de maior intensidade, e ocorrência numa faixa de temperatura e frequência mais estreita que a relaxação $\beta$. Verifica-se mais detalhadamente nos gráficos isocrônicos as duas relaxações e o seu deslocamento para as frequências maiores com o aumento da temperatura, devido à maior mobilidade das cadeias com o aquecimento.

Observa-se ainda que, a partir de $40^{\circ} \mathrm{C}$, a relaxação $\alpha$ apresenta uma separação em duas partes, sendo melhor visualizada em um gráfico das medidas de relaxação dielétrica em que a temperatura é mantida constante (isotérmico), apresentado na Figura 6. Este comportamento inesperado pode ser justificado pela característica bimodal do $\mathrm{PHB}^{[8-11]}$, onde a partir de $40^{\circ} \mathrm{C}$, as relaxações da fração oligomérica se distinguem da fração de maior massa molar. Na Figura 7 é apresentado o gráfico de $\mathrm{F}_{\max }$ vs. o inverso da temperatura, em frequência constante. Percebe-se claramente que as duas relaxações não pertencem ao mesmo processo, possuindo comportamentos distintos, com a relaxação denominada $\alpha_{1}$ apresentando um comportamento de Vogel-Fulcher, característico deste tipo de relaxação ${ }^{[8-11]}$.

As Figuras 8 e 9 apresentam a representação gráfica e a representação gráfica isocrônica de relaxação dielétrica do PLA, respectivamente. Estas medidas foram realizadas num filme prensado a quente.

Foram verificadas para o PLA duas relaxações: uma relaxação $\beta$ correspondente ao movimento das cadeias em poliésteres (também presente no PHB e no PCL), de formato largo e pouco intenso, que inicia ao redor de $-50^{\circ} \mathrm{C}$; e uma relaxação $\alpha$, intensa e mais estreita, característica da temperatura de transição vítrea deste material, que se inicia em aproximadamente $95^{\circ} \mathrm{C}$. Observa-se ainda que a intensidade da relaxação $\alpha$ tende a diminuir com o aumento

Tabela 2. Valores de DSC encontrados para o PHB, PLA e suas misturas.

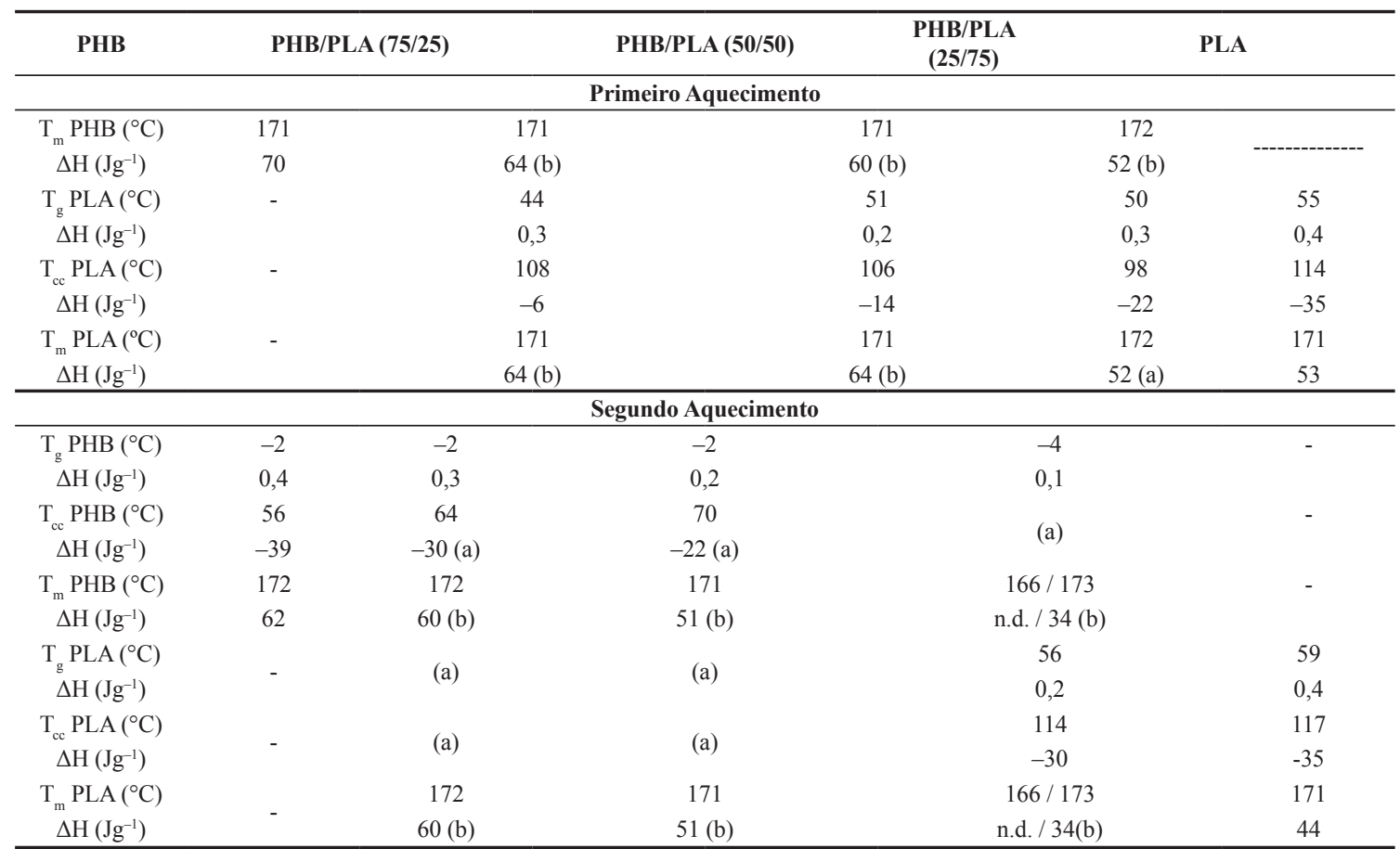

n.d.: não detectada; (a): valores relativos, devido a sobreposição da temperatura de cristalização a frio do PHB, temperatura de transição vítrea do PLA e temperatura de cristalização a frio do PLA; (b): valor relativo devido a sobreposição da temperatura de fusão do PHB e temperatura de fusão do PLA. 


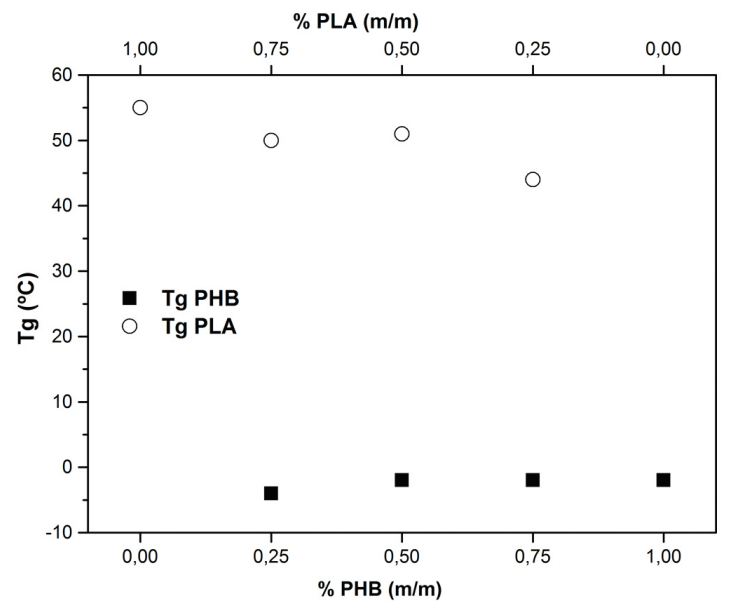

Figura 3. Gráfico dos valores da Tg do PHB e do PLA em suas misturas.

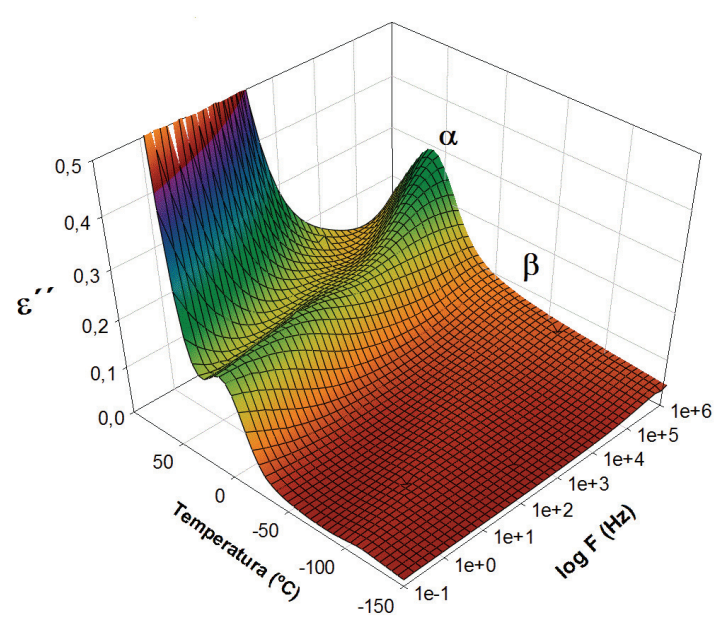

Figura 4. Medidas de relaxação dielétrica complexa para o PHB.

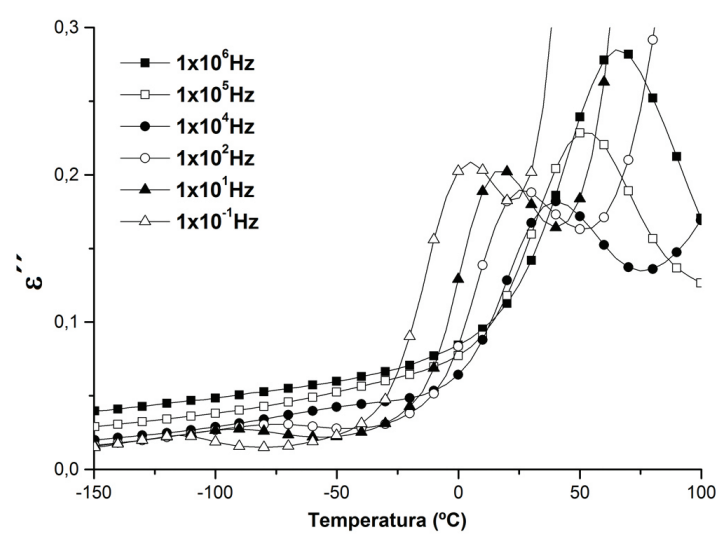

Figura 5. Medidas isocrônicas de relaxação dielétrica complexa para o PHB não processado.

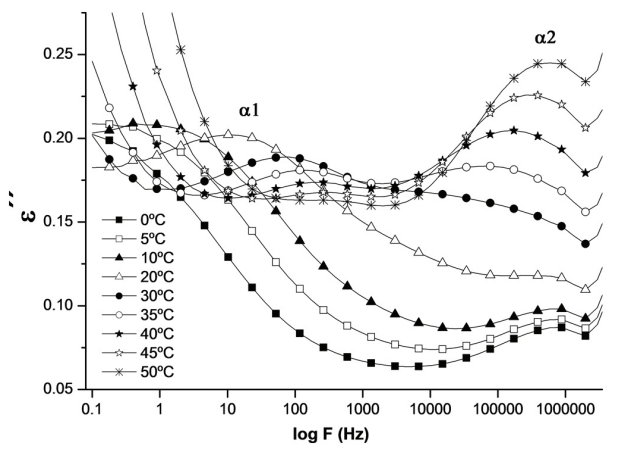

Figura 6. Medidas de relaxação dielétrica isotérmica (relaxação $\alpha$ ) do PHB.

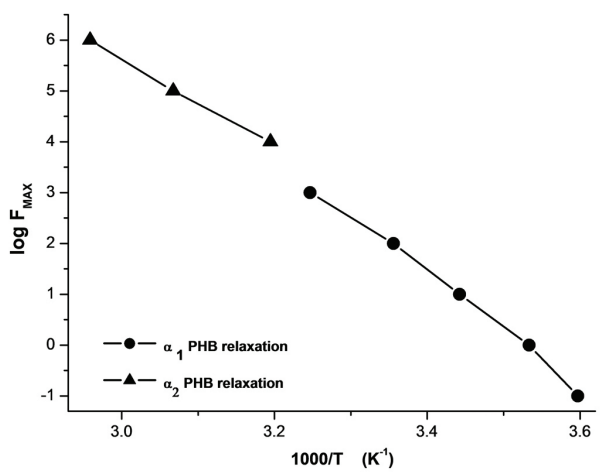

Figura 7. Gráfico comparativo das relaxações $\alpha$ do PHB.

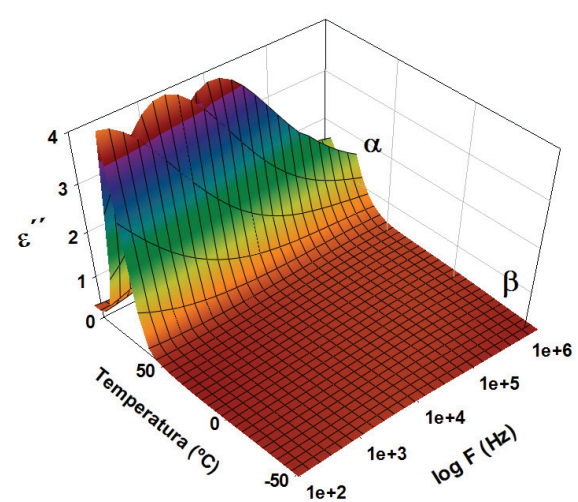

Figura 8. Medidas de relaxação dielétrica complexa do PLA.

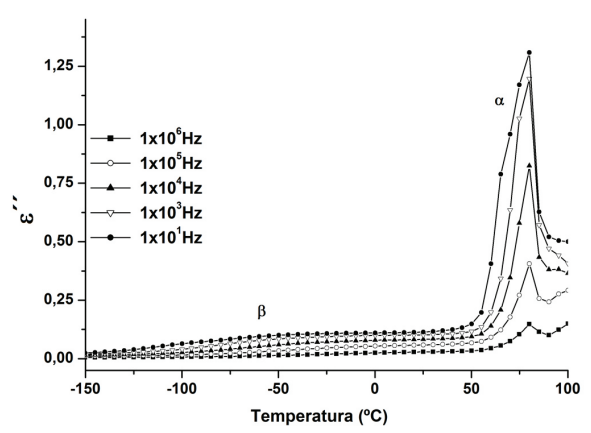

Figura 9. Medidas isocrônicas de relaxação dielétrica complexa para o PLA. 
da temperatura. Este efeito é explicado pela cristalização do material e perda de mobilidade, levando a uma redução na intensidade da relaxação $\alpha$.

A Figura 10a-c apresenta medidas de relaxação dielétrica complexa para as misturas PHB/PLA (75/25), $(50 / 50)$ e (25/75). A Figura 11 apresenta um gráfico comparativo das relaxações dielétricas de todas as amostras estudadas na frequência de $10 \mathrm{~Hz}$. Todas as medidas foram realizadas em filmes prensados a quente. As misturas de PHB e PLA apresentam três relaxações distintas: uma relaxação a partir de $-50^{\circ} \mathrm{C}$, que caracteriza o acoplamento das relaxações $\beta$ do PHB e do PLA; um processo a partir de $0^{\circ} \mathrm{C}$ pertencente a relaxação $\alpha$ do $\mathrm{PHB}$ e que determina a sua transição vítrea; e um processo iniciando em $50^{\circ} \mathrm{C}$, pertencente à relaxação $\alpha$ do PLA e sua transição vítrea.

A sobreposição das relaxações $\beta$ pode ser justificada no motivo pelo qual este processo ocorre: de movimentos locais de grupos carboxílicos em poliésteres. Como tanto o PHB como o PLA pertencem a esta classe de polímeros, e possuem estruturas relativamente parecidas, ambos
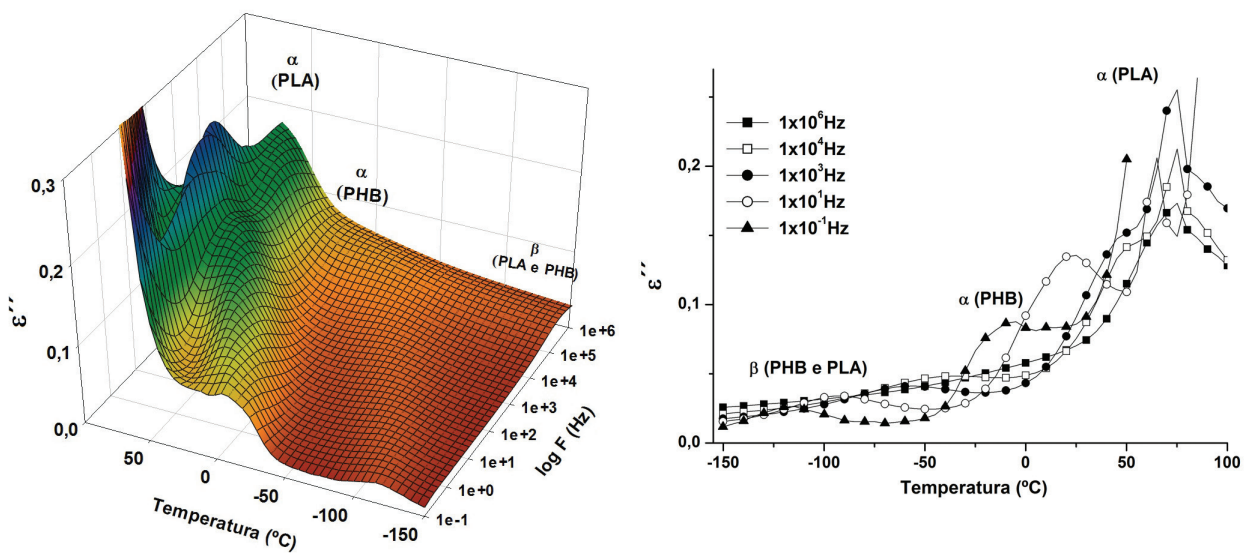

(a)
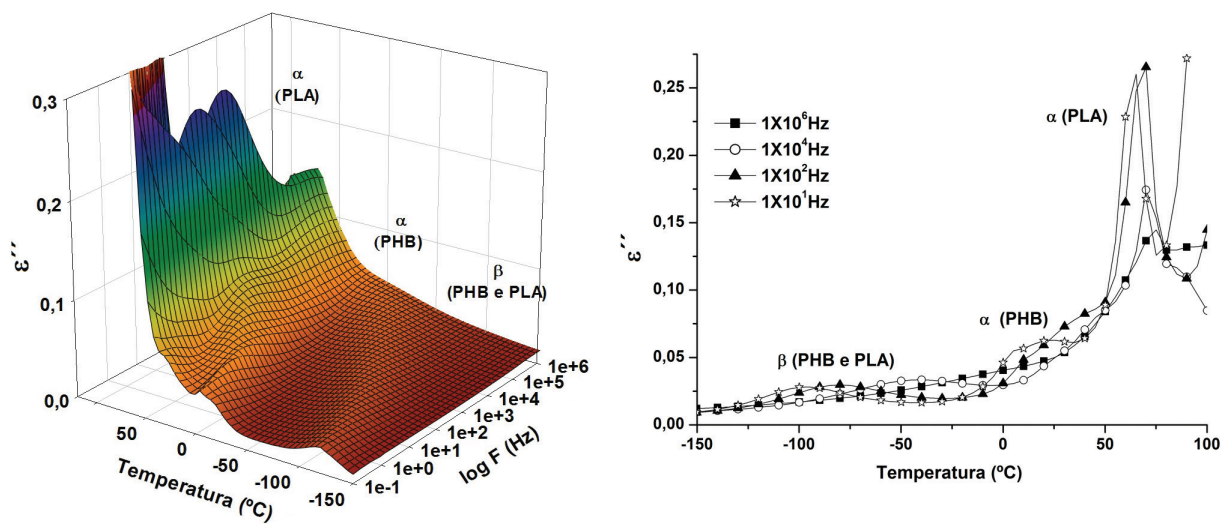

(b)
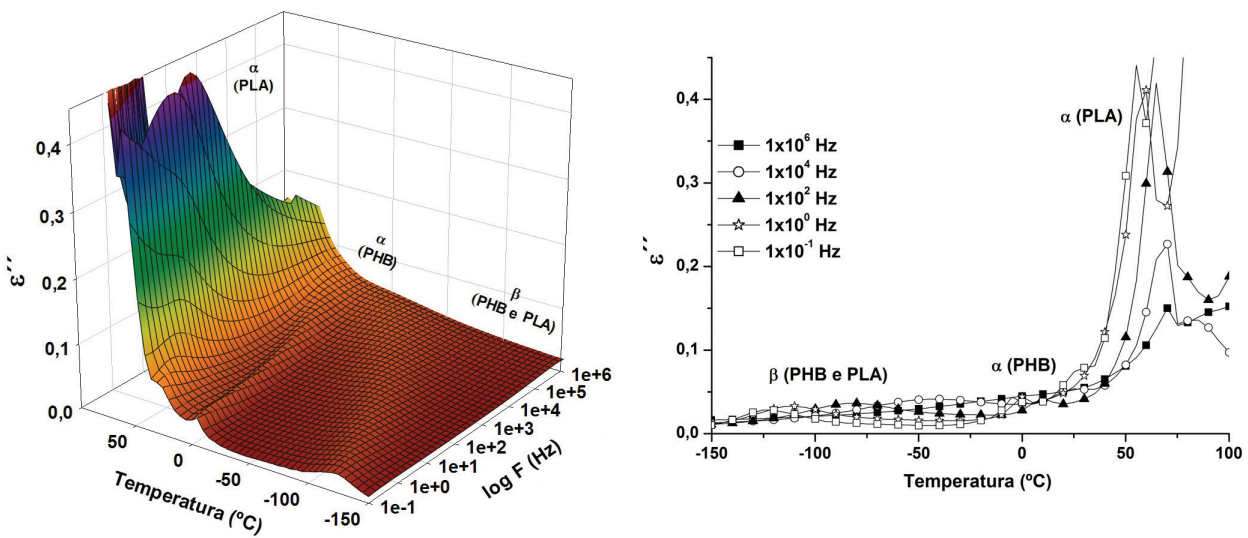

(c)

Figura 10. Medidas de relaxação dielétrica complexa para a mistura (a) PHB/PLA(75/25); (b) PHB/PLA(50/50); e (c) PHB/PLA(25/75). (À direita: medidas isocronais). 


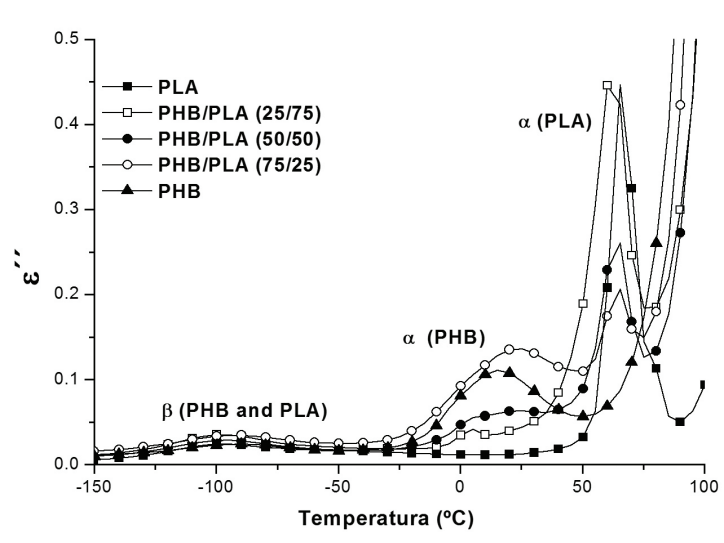

Figura 11. Medidas de relaxação dielétrica complexa para o PHB, PLA e suas misturas numa frequência de $10 \mathrm{~Hz}$.

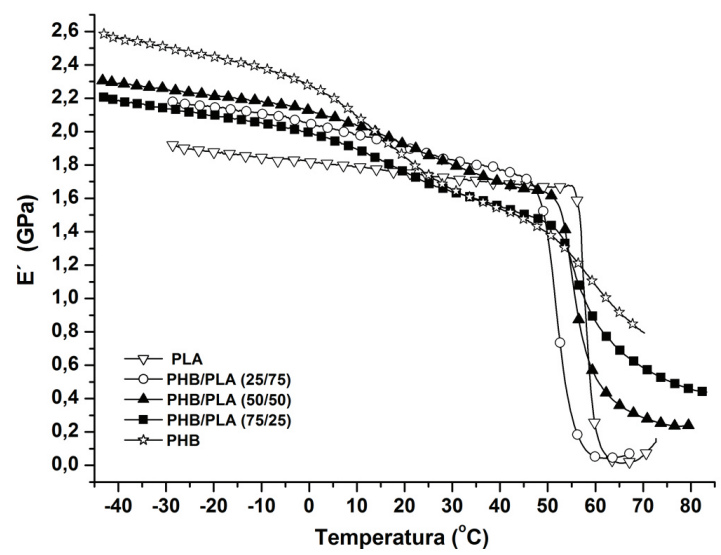

Figura 12. Resultados de DMA ( $E^{\prime}$ em função da temperatura) dos homopolímeros PHB, PLA e suas misturas.

apresentam este processo na mesma faixa de temperatura. Os processos possuem intensidades similares e grande amplitude, favorecendo a sobreposição.

As relaxações $\alpha$ do PHB e PLA nas misturas, pertencentes às suas respectivas temperaturas de transição vítrea, são independentes, situando-se na mesma região da temperatura de transição vítrea de cada polímero puro, e são proporcionais à quantidade de cada um dos polímeros em cada blenda. Este comportamento independente de cada polímero leva à conclusão que todas as misturas de PHB e PLA são imiscíveis, não ocorrendo interação entre os seus componentes. Na Figura 10a-c, pode-se observar o deslocamento da Tg do PLA nas misturas com PHB para temperaturas menores, devido à miscibilidade do PLA com a fração de baixa massa molar do $\mathrm{PHB}^{[21]}$.

\subsection{Análises Dinâmico Mecânicas (DMA)}

Foram realizados experimentos de DMA a partir de $-40^{\circ} \mathrm{C}$ a $100^{\circ} \mathrm{C}$. As Figuras 12 e 13 apresentam os resultados de $\varepsilon^{\prime}$ em função da temperatura e da $\tan \delta$ em função da temperatura, respectivamente. Observa-se o máximo referente à relaxação $\alpha$ do $\mathrm{PHB}$ próximo a $0^{\circ} \mathrm{C}$, enquanto a relaxação $\alpha$ do PLAé detectada em aproximadamente $60^{\circ} \mathrm{C}$. Nas misturas, verifica-se a presença de ambas as relaxações,

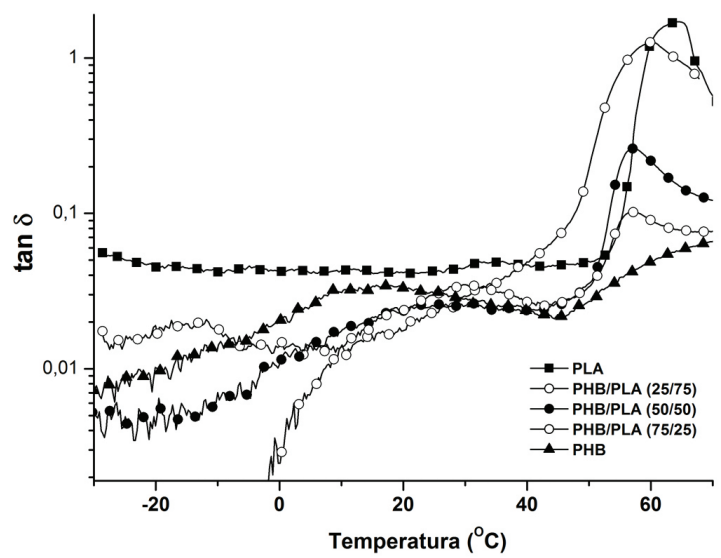

Figura 13. Resultados de DMA ( $\tan \delta$ em função da temperatura) dos homopolímeros PHB, PLA e suas misturas.

porém com um deslocamento da relaxação $\alpha$ do PLA para menores temperaturas, sugerindo a miscibilidade parcial da mistura. Verificou-se ainda uma redução na rigidez das misturas, em relação ao $\mathrm{PHB}$ puro. Porém, esta redução não foi tão significativa ao aumentar a porcentagem de PLA de $25 \%$ para $50 \%$ e $75 \%$.

\section{Conclusões}

As misturas de PHB e PLA são imiscíveis, apresentando duas temperaturas de transição vítrea e dois pontos de fusão em todas as suas misturas. Porém, a presença de uma fração de baixa massa molar no PHB influencia de maneira decisiva o comportamento deste homopolímero, gerando uma "pseudo-cristalização" a partir de $40^{\circ} \mathrm{C}$, assim como uma miscibilidade parcial de misturas de PHB e PLA. Esta miscibilidade parcial pode ser verificada pela redução na temperatura de transição vítrea proporcional à quantidade de PHB nas misturas PHB/PLA.

\section{Agradecimentos}

Os autores agradecem à PHB Industrial pelo fornecimento dos polímeros, e ao CNPq e FAPESC pelos auxílios financeiros.

\section{Referências}

1. Howells, E. R. (1982). Opportunities in biotechnology for the chemical industry. Chemistry \& Industry, 8, 508-511.

2. Platt, D. K. (2011). Biodegradable polymers: market report. New York: John-Wiley.

3. Kawaguchi, Y., \& Doi, Y. (1992). Kinetics and mechanism of synthesis and degradation of poly(3-hydroxybutyrate) in Alcaligenes eutrophus. Macromolecules, 25(9), 2324-2329. http://dx.doi.org/10.1021/ma00035a007.

4. Chen, G. (2010). Plastics from bacteria: natural functions and applications. Berlin: Springer-Verlag. http://dx.doi. org/10.1007/978-3-642-03287-5

5. Yokouchi, M., Chatani, Y., Tadokoro, H., Teranishi, K., \& Tani, H. (1973). Structural studies of polyesters: 5. Molecular and crystal structures of optically active and racemic poly (ß-hydroxybutyrate). Polymer, 14(6), 267-272. http://dx.doi. org/10.1016/0032-3861(73)90087-6. 
6. Skrbic, Z., \& Divjakovic, V. (1996). Temperature influence on changes of parameters of the unit cell of biopolymer PHB. Polymer, 37(3), 505-507. http://dx.doi. org/10.1016/0032-3861(96)82922-3.

7. Sato, H., Ando, Y., Mitomo, H., \& Ozaki, Y. (2011). Infrared spectroscopy and X-ray diffraction studies of thermal behavior and lamella structures of Poly(3-hydroxybutyrate- $c o$ -3-hydroxyvalerate) (P(HB- co-HV)) with PHB-Type crystal structure and PHV-Type crystal structure. Macromolecules, 44(8), 2829-2837. http://dx.doi.org/10.1021/ma102723n.

8. Pratt, G. J., \& Smith, M. J. A. (1997). Dielectric relaxation spectroscopy of a poly- $\beta$-hydroxybutyrate homopolymer. European Polymer Journal, 33(6), 857-861. http://dx.doi. org/10.1016/S0014-3057(96)00285-6.

9. Sics, I., Tupureina, V., Kalnins, M., Ezquerra, T. A., \& Baltá-Calleja, F. J. (1998). Dielectric relaxation of poly-( $\beta$-hydroxybutyrate) relating to microstructure. Journal of Macromolecular Science, B37(6), 851-862. http://dx.doi. org/10.1080/00222349808212421.

10. Hanafy, T. A., Elbanna, K., El-Sayed, S., \& Hassen, A. (2011). Dielectric relaxation analysis of biopolymer poly(3-hydroxybutyrate). Journal of Applied Polymer Science, 121(6), 3306-3313. http://dx.doi.org/10.1002/app.33950.

11. Pachekoski, W. M., Dalmolin, C., \& Agnelli, J. A. M. (2013). The influence of the industrial processing on the degradation of poly(hidroxybutyrate) - PHB. Materials Research, 16(2), 327. http://dx.doi.org/10.1590/S1516-14392012005000180.

12. Datta, R., Tsai, S., Bonsignore, P., Moon, S., \& Frank, J. (1995). Technological and economic potential of poly(lactic acid) and lactic acid derivatives. FEMS Microbiology Reviews, 16(2-3), 221-231. http://dx.doi.org/10.1111/j.1574-6976.1995. tb00168.x.

13. Ren, J. (2011). Biodegradable poly(lactic acid): synthesis, modification, processing and application. New York: Springer. http://dx.doi.org/10.1007/978-3-642-17596-1.

14. AURAS, R. A., LIM, L., SELKE, S. E. M., \& TJUDI, H. (2010). Poly(lactic acid): synthesis, structures, properties, processing, and applications. New York: John-Wiley. http:// dx.doi.org/10.1002/9780470649848.

15. Seal, B. L., Otero, T. C., \& Panitch, A. (2001). Polymeric biomaterials for tissue and organ regeneration. Materials Science and Engineering, 34(4-5), 147-230. http://dx.doi. org/10.1016/S0927-796X(01)00035-3.

16. Zhang, L., Xiong, C., \& Deng, X. (1995). Biodegradable polyester blends for biomedical application. Journal of Applied Polymer Science, 56(1), 103-112. http://dx.doi. org/10.1002/app.1995.070560114.

17. Domb, A. J., \& Kumar, N. (2011). Biodegradable polymers in clinical use and clinical development. New York: John-Wiley. http://dx.doi.org/10.1002/9781118015810.

18. Garlotta, D. (2001). A literature review of poly(lactic acid). Journal of Polymers and the Environment, 9(2), 63-84.
19. Henton, D. E., Gruber, P. G., Lunt, J., \& Randall, J. (2005). Polylactic acid technology. In A. K. Mohanty, \& L. Dzral (Eds.), Natural fibers, biopolymers, and biocomposites (pp. 527-578). New York: CRC Press. http://dx.doi. org/10.1201/9780203508206.ch16.

20. Zhang, L., Xiong, C., \& Deng, X. (1996). Miscibility, crystallization and morphology of poly( $\beta$-hydroxybutyrate)/ poly(d,1-lactide) blends. Polymer, 37(2), 235-241. http:// dx.doi.org/10.1016/0032-3861(96)81093-7.

21. Koyama, N., \& Doi, Y.(1995). Morphology and biodegradability of a binary blend of poly((R)-3-hydroxybutyric acid) and poly((R,S)-lactic acid). Canadian Journal of Microbiology, 41(13), 316-322. http://dx.doi.org/10.1139/m95-203. PMid:7606667

22. Blümm, E., \& Owen, E. J. (1995). Miscibility, crystallization and melting of poly(3-hydroxybutyrate)/ poly(l-lactide) blends. Polymer, 36(21), 4077-4081. http://dx.doi. org/10.1016/0032-3861(95)90987-D.

23. Ohkoshi, I., Abe, H., \& Doi, Y. (2000). Miscibility and solid-state structures for blends of poly[(S)-lactide] with atactic poly[(R,S)-3-hydroxybutyrate]. Polymer, 41(15), 5985-5992. http://dx.doi.org/10.1016/S0032-3861(99)00781-8.

24. Gerard, T., \& Budtova, T. (2012). Morphology and molten-state rheology of polylactide and polyhydroxyalkanoate blends. European Polymer Journal, 48(6), 1110-1117. http://dx.doi. org/10.1016/j.eurpolymj.2012.03.015.

25. Ficha Técnica de caracterização poli (hidroxibutirato) lote 60-3. (2015, Fevereiro 3). Recuperado em http://www. biocycle.com.br/site.htm.

26. Poly latic acid Lacea H-100J Datasheet. (2015, Fevereiro 3). Recuperado em http://plastics.ulprospector.com/pt/datasheet/ e52212/lacea-h-100j-stretched.

27. Abdelwahab, M. A., Flynn, A., Chiou, B.-S., Imam, S., Orts, W., \& Chiellini, E. (2012). Thermal, mechanical and morphological characterization of plasticized PLA-PHB blends. Polymer Degradation and Stability, 97(9), 1822-1828. http://dx.doi.org/10.1016/j.polymdegradstab.2012.05.036.

28. Zhang, M., \& Thomas, N. L. (2011). Blending polylactic acid with polyhydroxybutyrate: the effect on thermal, mechanical, and biodegradation properties. Advances in Polymer Technology, 30(2), 67-79. http://dx.doi.org/10.1002/ adv.20235.

29. Chang, L., \& Woo, E. M. (2011). Effects of molten poly(3-hydroxybutyrate) on crystalline morphology in stereocomplex of poly(L-lactic acid) with poly(D-lactic acid). Polymer, 52(1), 68-76. http://dx.doi.org/10.1016/j. polymer.2010.11.028.

Enviado: Out. 12, 2013

Reenviado: Maio 24, 2014

Aceito: Ago. 04, 2014 